\title{
Multi-minicore disease and atypical periodic paralysis associated with novel mutations in the skeletal muscle ryanodine receptor $(R Y R 1)$ gene
}

\author{
Haiyan Zhou ${ }^{a}$, Suzanne Lillis ${ }^{b}$, Ryan E. Loy ${ }^{c}$, Farshid Ghassemi ${ }^{d}$, Michael R. Rose ${ }^{e}$, Fiona \\ Norwood $^{\mathrm{e}}$, Kerry Mills ${ }^{\dagger}$, Safa Al-Sarraj ${ }^{\mathrm{g}}$, Russell J.M. Lane ${ }^{\mathrm{h}}$, Lucy Feng ${ }^{\mathrm{a}}$, Emma Matthews , $^{\mathrm{i}}$, \\ Caroline A. Sewryj, Stephen Abbs ${ }^{b}$, Stefan Buk ${ }^{g}$, Michael Hannai, Susan Treves ${ }^{k}$, Robert T. \\ Dirksen $^{c}$, Gerhard Meissner ${ }^{d}$, Francesco Muntoni ${ }^{\mathrm{a}}$, and Heinz Jungbluth ${ }^{1, m,{ }^{*}}$
}

a Dubowitz Neuromuscular Centre, UCL Institute of Child Health \& Great Ormond Street Hospital for Children, London, UK ${ }^{b}$ DNA Laboratory, GSTS Pathology, Guy's Hospital, London, UK ${ }^{c}$ University of Rochester Medical Center, Department of Pharmacology and Physiology, Rochester, New York, USA d University of North Carolina, Department of Biochemistry and Biophysics, Chapel Hill, North Carolina, USA ${ }^{e}$ Department of Neurology, King's College Hospital, London, UK ${ }^{f}$ Department of Neurophysiology, King's College Hospital, London, UK ${ }^{9}$ Department of Clinical Neuropathology, King's College Hospital, London, UK ${ }^{\mathrm{h}}$ Department of Clinical Neurosciences, Charing Cross Hospital, London, UK ' MRC Centre for Neuromuscular Diseases, UCL Institute of Neurology, National Hospital for Neurology and Neurosurgery, Queen Square, London, UK ${ }^{\mathrm{j}}$ Centre for Inherited Neuromuscular Disorders, RJAH Orthopaedic Hospital, Oswestry, UK k Departments of Anaesthesia and Research, Basel University Hospital, 4031 Basel, Switzerland ' Department of Paediatric Neurology, Evelina Children's Hospital, St. Thomas' Hospital, London, UK ${ }^{m}$ Clinical Neuroscience Division, King's College, London, UK

\begin{abstract}
The skeletal muscle ryanodine receptor plays a crucial role in excitation-contraction (EC) coupling and is implicated in various congenital myopathies. The periodic paralyses are a heterogeneous, dominantly inherited group of conditions mainly associated with mutations in the $S C N 4 A$ and the CACNAIS genes. The interaction between RyR1 and DHPR proteins underlies depolarization-induced $\mathrm{Ca}^{2+}$ release during EC coupling in skeletal muscle. We report a 35-yearold woman presenting with signs and symptoms of a congenital myopathy at birth and repeated episodes of generalized, atypical normokalaemic paralysis in her late teens. Genetic studies of this patient revealed three heterozygous RYR1 substitutions (p.Arg2241X, p.Asp708Asn and p.Arg2939Lys) associated with marked reduction of the RyR1 protein and abnormal DHPR distribution. We conclude that $R Y R I$ mutations may give rise to both myopathies and atypical periodic paralysis, and $R Y R I$ mutations may underlie other unresolved cases of periodic paralysis with unusual features.
\end{abstract}

\section{Keywords}

Multi-minicore disease (MmD); Periodic paralysis; Excitation-contraction coupling (ECC); Skeletal muscle ryanodine receptor (RYR1); gene

\footnotetext{
(C) 2010 Elsevier B.V. All rights reserved.

*Corresponding author. Address: Clinical Neuroscience Division, King's College London, and Department of Paediatric Neurology, Evelina Children's Hospital, Guy's \& St. Thomas' NHS Foundation Trust, Lambeth Palace Road, London SE1 7EH, UK. Heinz.Jungbluth@gstt.nhs.uk (H. Jungbluth).
} 


\section{Introduction}

The skeletal muscle ryanodine receptor (RYR1) gene on chromosome 19q13.1 encodes the principal sarcoplasmic reticulum (SR) calcium release channel (RyR1), which plays a crucial role in excitation- contraction (EC) coupling by releasing calcium from the SR following muscle activation. RYRI mutations have been implicated in a wide range of conditions (for review [1]). Among these are malignant hyperthermia susceptibility (MHS) [2] and various congenital myopathies including "core myopathies" with central cores (Central Core Disease, CCD) [3], multiple cores (Multi-minicore disease, MmD) with external ophthalmoplegia [4] and, rarely, Centronuclear Myopathy (CNM) [5]. Whilst MHS is dominantly inherited, CCD involves both dominant and recessive inheritance. $\mathrm{MmD}$ with external ophthalmoplegia is associated with recessive inheritance and quantitative defects of RyR1 protein expression, due to epigenetic allele silencing [6] or compound heterozygosity for RYRI null and missense mutations [7,8].

The periodic paralyses are a genetically heterogeneous group of rare, dominantly inherited conditions characterized by episodic weakness and divided into hyperkalaemic periodic paralysis (HYPP, OMIM 170500), mainly caused by SCN4A missense mutations $[9,10]$, and hypokalaemic periodic paralysis (HOKPP, OMIM170400), predominantly associated with mutations in the CACNAIS gene [11], which encodes the $\alpha 1 S$ subunit of the dihydropyridine receptor (DHPR) or L-type calcium channel in skeletal muscle. The close functional relationship between the DHPR and RyR1 is reflected by their co-localization and linkage within the triad [12], convergence of associated clinical phenotypes, and findings of abolished EC coupling in animal models lacking either of the two proteins $[13,14]$. Whilst MHS has been reported in one French family harbouring a dominant CACNAIS mutation [15], prior studies failed to provide a definitive link between $R Y R I$ mutations and periodic paralysis [16].

We report a 35-year-old patient with Multi-minicore disease (MmD) with external ophthalmoplegia and additional episodes of atypical periodic paralysis associated with compound heterozygosity for a nonsense and missense mutations in $R Y R$. Detailed neurophysiological and immunohistochemical studies in this patient suggested a dramatic reduction in RyR1 protein expression with resulting marked disruption of the EC coupling machinery.

\section{Patient and methods}

\subsection{Patient}

This 35-year-old female was the oldest child of a healthy non-consanguineous couple of Irish ancestry. Two younger brothers were healthy and her deceased sister had suffered from psychiatric problems. There was no preceding family history of neuromuscular disorders. She presented at birth with hypotonia, respiratory impairment and feeding difficulties following an uneventful pregnancy and normal vaginal delivery at term. Her motor development was delayed and she was unable to walk before 30 months of age. By age 12 she had difficulties rising from supine or squatting positions, managing stairs, was unable to hop or jump, and suffered frequent falls. From the age of 17 years, she had several episodes of spontaneous hip dislocations. Following birth of her first child at the age of 26 years, she experienced increasing problems with reduced walking distance and difficulties with both overhead and fine manual tasks. Exercise-induced myalgia and muscle stiffness prompted by cold, sometimes with weakness affecting the hands, were frequent complaints. There was no cognitive, bulbar or cardiorespiratory involvement. 
In addition, from the age of 18 she experienced repeated episodes of severe paralysis initially affecting the legs and then rapidly spreading to the upper limbs lasting up to several days. These episodes were typically preceded by a sensation of dizziness, facial and acral paraesthesia, temporal pain and a visual aura comprising flashing lights, blurred vision and diplopia. Respiratory, bulbar and sphincter functions were usually unimpaired. One episode each was triggered by immersion in a cold swimming pool and another followed administration of lignocaine for a minor surgical procedure, which also prompted marked hyperpyrexia. Her episodes improved following commencement of a potassium-sparing, high sodium diet.

She had four general anaesthetics for surgical procedures and did not experience any $\mathrm{MH}$ reaction. Whilst only non-triggering anaesthetic agents were used for the last two anaesthetics, no further details could be obtained regarding the first two anaesthetics administered many years ago in different hospitals. She did not have any in vitro contracture testing to formally assess for malignant hyperthermia susceptibility (MHS).

On a recent examination at 32 years of age, there was facial weakness with incomplete eye closure and limitation of extraocular movements mainly on abduction and upgaze. She had slight nasal escape on speaking and a high arched palate, with weak palatal movements but no dysphonia. There was wasting of the deltoids and distal muscles in the upper limb. There was marked axial weakness on trunk and neck flexion (MRC2), moderate limb girdle weakness (MRC3/4) more pronounced in the shoulder than the hip girdle muscles with additional distal weakness in the upper limbs pronounced in the first dorsal interosseoi (MRC3). There was distal joint hyperlaxity, particularly affecting the hands. She had a hyperlordotic stance but there was no kyphoscoliosis. No action or percussion myotonia was evident. Forced vital capacity was 3.05 1. ECG was normal and an echocardiograph showed no structural or functional abnormalities.

Muscle MRI of the lower limb performed as previously described [17] showed diffuse involvement with relative sparing of the rectus femoris compared to the vasti, and the anterior compartment compared to the gastrocnemius and the soleus within the lower leg, a pattern suggestive of involvement of the RYRI gene [4,17].

Concentric needle EMG showed myopathic changes in the left biceps and suggested chronic neurogenic changes in the small hand muscles; there was no electrophysiological evidence of myotonia. Cold immersion testing prompted profound weakness of the hands but normally preserved CMAP responses in the right ADM both pre- and post-cooling, suggesting cold-induced E-C uncoupling; EMG of the cooled small hand muscles showed fibrillations and fasciculations with relatively large motor units. An exercise test for periodic paralysis [18] performed at 21 years was within normal limits. The long exercise test performed at the age of 30 years was negative but demonstrated an unexpected increase in CMAP amplitude and area post-exercise. Oral potassium loading resulted in profound paralysis more than $12 \mathrm{~h}$ after the loading but potassium levels were normal when measured during the episode. A forearm ischaemic exercise test and routine laboratory investigations including CK were normal.

\subsection{Molecular genetic analysis}

$S C N 4 A$ and $C L C N 1$ mutations were excluded by genomic DNA sequencing. The entire coding regions (exon 1-106) of the RYRl gene including splice sites were screened at both genomic DNA and RNA levels. The entire coding sequence of the CACNAIS (NM_000069.2) and CACNB1 (NM_000723.3) gene were also screened by using cDNA synthesized from skeletal muscle as template. 


\subsection{Muscle biopsy}

The patient had two muscle biopsies in early childhood and in her teens which suggested a diagnosis of MmD. However, on review of her earlier biopsies appearances were less suggestive of typical $\mathrm{MmD}$ associated with mutations in the selenoprotein $\mathrm{N}$ (SEPN1) gene $[19,20]$ and because of the diagnostic uncertainty repeat muscle biopsy samples were obtained from the quadriceps (rectus femoris) at 30 years of age.

Cryostat sections were stained with haematoxylin and eosin (H\&E), periodic acid Schiff (PAS), nicotinamide dehydrogenase tetrazolium reductase (NADH-TR), succinate dehydrogenase (SDH) and cytochrome oxidase (COX), according to standard procedures [21]. Immunohistochemistry was done by double staining with primary antibodies: mouse anti-RyR1 monoclonal antibody (1:500, Abcam) and goat anti-DHPR polyclonal antibody (1:1000, Santa Cruz). Sections were incubated at room temperature for $1 \mathrm{~h}$, and then washed in phosphate buffered saline (PBS) and incubated $30 \mathrm{~min}$ at room temperature with antimouse biotinylated secondary antibody (1:200, Amersham). After further washes in PBS, sections were incubated at room temperature for $30 \mathrm{~min}$ with streptavidin-conjugated Alexa-488 (1:1000, Molecular Probes) and donkey anti-goat Alexa-594 (1:1000, Molecular Probes). Sections were viewed with a Leica fluorescent DMR microscope (Leica, Germany). Images were captured digitally using Metamorph software (Universal Imaging Inc.) with auto-exposure settings. To further investigate the distribution of the DHPR and RyR1 proteins, sections were also examined by high magnification confocal microscope (Zeiss LSM 710, Germany).

Western blotting of RyR1 protein extracted from patient's muscle, and routine histopathological and immunohistochemical staining were performed as described previously [21,22]. Band densities are relative to the respective desmin band, used as a control of protein loading. The densitometric analyses were performed using Image $\mathbf{J}$ software.

\subsection{RyR1 functional studies}

To determine the functional consequences of the p.Arg2939Lys mutation more directly, RyR1-R2939K was constructed, expressed in HEK 293 cells, and crude membrane fractions were prepared as previously described [23]. $\left[{ }^{3} \mathrm{H}\right]$ Ryanodine binding was used as a measurement of RyR1 activity [24]. Specific $\left[{ }^{3} \mathrm{H}\right]$ ryanodine binding to membrane fractions was determined for $20 \mathrm{~h}$ at $24^{\circ} \mathrm{C}$ in $0.25 \mathrm{M} \mathrm{KCl}, 20 \mathrm{mM}$ imidazole, $\mathrm{pH} 7$ solution containing $5 \mathrm{mM} \mathrm{Mg}^{2+}, 5 \mathrm{mM}$ AMPPCP (a nonhydrozyableATP analog), $5 \mathrm{mM}$ glutathione (reduced) to mimick physiological conditions, $1.5-2.5 \mathrm{nM}\left[{ }^{3} \mathrm{H}\right]$ ryanodine and the indicated concentrations of caffeine and free $\mathrm{Ca}^{2+}[23]$.

Voltage clamp studies were performed following expression of wild-type and mutant RyR1R2939K in RyR1-null myotubes from dyspedic mice as described previously [25]. The whole-cell perforated voltage clamp technique was used to simultaneously measure L-type $\mathrm{Ca}^{2+}$ currents (L-currents) and intracellular $\mathrm{Ca}^{2+}$ transients in expressing myotubes as described previously [26].

\section{Results}

\subsection{Molecular genetic analysis of the RYR1 gene}

An apparently homozygous nucleotide variation of c.8816G>A was identified in exon 57 of the RYRI cDNA synthesized from patient's skeletal muscle biopsy [22]. This maternally inherited missense mutation affects the last nucleotide of exon 57 and caused a substitution of p.Arg2939Lys. The p.Arg2939Lys mutation was not found neither in >200 normal 
control chromosomes from Caucasian populations nor among more than 50 previously reported MHS/CCD individuals of both Caucasian and Asian origin in whom the entire RYRl coding sequence had been analyzed $[27,28]$. Furthermore, the c. $8816 \mathrm{G}>\mathrm{A}$ nucleotide change, apparently homozygous in muscle, was heterozygous at the genomic DNA level (Fig. 1), a discrepancy previously reported in $\mathrm{MmD}$ with ophthalmoplegia [4,6,8]. The substituted arginine residue is strictly conserved in all three RyR isoforms and throughout species (Fig. 2). Further sequencing of the RYRI gene from lymphocyte-derived gDNA from this patient eventually revealed an additional heterozygous c.6721 C > T substitution resulting in a p.Arg2241X stop mutation, and an additional heterozygous c.2122G>A change resulting in a p.Asp708Asn substitution on the same allele. mRNA produced from the allele bearing these mutations was likely affected by nonsense-mediated decay, which would account for the inability to amplify this allele from cDNA. In particular, the heterozygous c. $2122 \mathrm{G}>\mathrm{A}$ (p.Asp708Asn) substitution was undetectable at RNA level suggesting that both this substitution and p.Arg2241X originate from the same allele that was not transcribed. The p.Asp708Asn substitution changes a charged polar to an uncharged polar amino acid and concerns a highly conserved residue. However, the fact that this substitution was not transcribed at mRNA level makes a pathogenetic role unlikely. Maternal inheritance was excluded for the c.6721 C>T (p.Arg2241X) and the c.2122G >A (p.Asp708Asn) substitutions but no DNA was available from her father and siblings for further segregation studies.

\subsection{Light microscopy, immunohistochemistry and Western blot analysis of the muscle biopsy}

Muscle biopsy performed at 30 years showed increased variability in fibre size and increase in internal nuclei in up to $25 \%$ of fibres. Internal nuclei were sometimes central and often multiple (Fig. 3A, a and c). There was no excess endomysial connective tissue. There was predominance of hypotrophic type 1 fibres (Fig. 3A, d). Core-like structures of varying size were apparent with oxidative enzyme stains (Fig. 3A, b) and confirmed on electron microscopy (Fig. 3A, e and f).

Double immunohistochemical labelling of control muscle with monoclonal antibodies to RyR1 and DHPR proteins demonstrated nearly identical distribution pattern of the two proteins as shown by the yellow staining in the merged image (Fig. 3B, f). However, in the patient's muscle reduced signal from the RyR1 protein (Fig. 3B, b and c) and abnormal accumulation of DHPR protein in a subpopulation of fibres (Fig. 3B, a and c) were observed. On further examination of biopsy sections by confocal microscope, unevenness of stain with the RyR1 antibody was noted in regions corresponding to areas of distinct DHPR aggregation (Fig. 4).

In order to quantify RyR1 protein in this patient we performed Western blot studies on patient's skeletal muscle. RyR1 protein expression levels assessed in Western blotting analysis revealed a marked reduction of RyR1 protein content in the skeletal muscle biopsy from the patient compared to that from a normal control individual (Fig. 3C). This is in keeping with what we have reported recently in other patients with $\mathrm{MmD}$ and ophthalmoplegia [22]. On densitometric analysis, there was only about $20 \%$ residual RyR1 protein expressed in skeletal muscle from the patient, less than expected due to nonsensemediated RNA decay of the allele harbouring the p.Arg2241X substitution alone.

\subsection{RyR1 functional studies}

$\left[{ }^{3} \mathrm{H}\right]$ Ryanodine binding is a sensitive method to evaluate regulation of RyR1 by its endogenous and exogenous effectors [24]. As shown in Fig. 5A, membrane fractions isolated from HEK 293 cells expressing wild type (WT) RyR1 and RyR1-R2939K showed a 
comparable bimodal $\mathrm{Ca}^{2+}$-dependence of $\left[{ }^{3} \mathrm{H}\right]$ ryanodine binding. We also probed the effects of caffeine, an exogenous ligand known to augment RyR1 activation [24]. Fig. 5B shows that caffeine increased to the same extent $\left[{ }^{3} \mathrm{H}\right]$ ryanodine binding to membranes containing WT-RyR1 and RyR1-R2939K. The results suggest that the R2435K mutation did not affect two characteristic functional properties of RyR 1 , as both $\mathrm{Ca}^{2+}$ dependence and activation by caffeine were not altered.

Since the R2939K mRNA was the only RYRI transcript detected in the skeletal muscle of the patient, we compared EC coupling in dyspedic myotubes following homozygous expression of either wild type RYRI or R2939K. The whole-cell perforated patch clamp technique was used to simultaneously assess the magnitude and voltage dependence of depolarization-induced $\mathrm{Ca}^{2+}$ release (upper traces in Fig. 6A; "orthograde" coupling) and Ltype $\mathrm{Ca}^{2+}$ current density (lower traces in Fig. 6A; "retrograde" coupling). Peak L-current density $\left(G_{\max }\right)$ and the voltage dependence of channel activation $\left(V_{\mathrm{G} 1 / 2}\right)$ were not significantly different between WT- and R2939K-expressing myotubes (Fig. 6B and Table $\mathrm{S} 1)$. Moreover, the magnitude $\left(F_{\max }\right)$ and voltage dependence $\left(V_{\mathrm{F} 1 / 2}\right)$ depolarizationinduced $\mathrm{Ca}^{2+}$ release (Fig. $6 \mathrm{C}$ and Table S1) and DHPR-RyR1 co-localization (Supplementary figure) were also not significantly different in WT- and R2939K-expressing myotubes. Thus, the results presented in Figs. 4 and 5 indicate that the conservative R2939K mutation identified in the patient does not directly alter RyR1 channel function.

\section{Discussion}

The distribution of wasting and weakness, histopathologic features and radiological findings on muscle MRI in the patient reported in this paper are identical to those of other patients with Multi-minicore disease $(\mathrm{MmD})$ with external ophthalmoplegia attributed to homozygosity [7], compound heterozygosity $[4,8]$ or monoallelic expression of heterozygous RYRI missense mutations due to haploinsufficiency associated with either epigenetic allele silencing [6] or nonsense-mediated RNA decay [8]. In addition, the patient in this report exhibited clinical features suggestive of atypical periodic paralysis, not previously reported to be associated with mutations in the RYRI gene.

Despite suggestive symptoms, the precise nature of the periodic paralysis in our patient was difficult to define and her presentation was clearly divergent from that seen in other forms of genetically resolved periodic paralysis. Although clinical features, in particular exertional myalgia, paralytic attacks precipitated by cold or $\mathrm{K}^{+}$-loading and improvement of symptoms with a kaliuretic, were most suggestive of a form of hyperkalaemic periodic paralysis (HYPP), there was no overt myotonia and the long and short exercise tests were negative. Most importantly, measurement of serum $\mathrm{K}^{+}$-levels during a paralysis attack induced by $\mathrm{K}^{+}$administration were normal, suggesting normokalaemic periodic paralysis, a variant of HYPP, by definition.

Regarding the mutations identified, several lines of evidence indicate their pathogenecity: (1) the c.6721 C>T substitution leads to a premature stop codon, (2) no polymorphisms have been previously reported at position 2939 and the amino acid residue is preserved across different RyR isoforms and species, (3) compound heterozygosity for RYRI nonsense and missense mutations corresponds to findings in other patients with $\mathrm{MmD}$ and external ophthalmoplegia [8], (4) the patient's clinical presentation matches that associated with monoallelic expression of paternally inherited missense mutations on a haploinsufficient background due to epigenetic silencing of the maternal allele [6] and (5) other potential causes of the periodic paralysis phenotype in this patient (e.g. mutations in CACNAIS and $S C N 4 A$ genes) were excluded. 
It seems unlikely that the co-occurrence of $\mathrm{MmD}$ and atypical periodic paralysis in the same patient might be attributed to "double-trouble" due to an additional mutation in an as yet unidentified gene, considering the combined clinical, neurophysiological and immunohistochemical evidence for the RyR1-R2939K mutation altering proper RyR1 longterm expression and stoichiometry of the EC coupling machinery in vivo. In particular, the combination of profound weakness but preserved compound muscle action potentials (CMAPs) in the hand muscles following cold immersion testing is unusual in the context of other forms of periodic paralysis and suggests a temperature dependent, functionally relevant disruption of EC coupling in the patient, in spite of normal calcium dependence of ryanodine binding (Fig. 5) and voltage-gated calcium release following transient expression in myotubes (Fig. 6). Altered DHPR-RYR1 stoichiometry in the patient's muscle is supported by reduced RyR1 expression (Fig. 3), as well as uneven and significant fiber-tofiber variability in RyR1/DHPR abnormal distribution (Figs. 3 and 4). Although episodes of atypical periodic paralysis have not been previously described in other cases of RYRIrelated $\mathrm{MmD}$ with external ophthalmoplegia, this might be partly explained by a relatively low penetrance of this particular trait. While our patient presented with features of a congenital myopathy at birth, paralytic episodes did not develop until age 19 years and responded favourably to commencement of a potassium sparing diet. Since many of the patients with $\mathrm{MmD}$ with external ophthalmoplegia reported to date were infants or young children [4], the periodic paralysis trait may not have developed at the time those cases were reported. In addition, despite the lack of previously reported paralytic episodes in $\mathrm{MmD}$ with external ophthalmoplegia, the marked sensitivity of muscle weakness to cold in these individuals [4] points to a possible continuum between the congenital myopathy phenotype and the manifestations of atypical periodic paralysis.

Our results indicate abnormal RyR1 protein expression and DHPR-RyR1 distribution in the quadriceps of the patient in vivo, but, rather unexpectedly, normal RyR1 function and RyR1/ DHPR co-localization in vitro following transient homozygous expression of R2939K in dyspedic myotubes. This apparent discrepancy could be explained by a slow time-dependent quantitative, rather than qualitative, effect of the mutation, expressed homozygously on a background of already reduced RyR1 protein due to nonsense-mediated RNA decay of the second allele harbouring an allelic $R Y R I$ nonsense mutation. Specifically, rather than disrupting intrinsic RyR1 channel activity, the homozygous p.Arg2939Lys mutation may alter the long-term stability of RyR1-DHPR interaction by affecting RyR1 junctional localization and/or degradation, but unfortunately we were unable to obtain more stable muscle cell lines to investigate this hypothesis further. Although the p.Arg2939Lys mutation is a relatively conservative substitution, the presence of a lysine residue at this site could potentially introduce a novel ubiquitination site that may alter the protein's long-term stability and/or localization [29]. An effect of the p.Arg2939Lys substitution on long-term protein stability is also supported by the observation of in vivo RyR1 protein reduction to only about $20 \%$ of the normal amount, much less than can be explained by nonsensemediated RNA decay of the allele harbouring the p.Arg2241X substitution alone. Impaired stability of the DHPR/RyR1 complex has also been implicated in the zebrafish relatively relaxed mutant, a model of $\mathrm{MmD}$ due to a nonsense mutation in the piscine fast muscle ryanodine receptor isoform ryrlb [13]. Zebrafish relatively relaxed mutants harbouring this mutation display similar findings of markedly reduced DHPR/RyR1 complexes associated with an E-C uncoupling defect, further supporting a causative role of the RYRI gene in the corresponding human phenotype. As evidenced by the asymptomatic carrier mother of the patient, heterozygosity for R2939K does not appear to be associated with a clinical phenotype.

It remains uncertain whether the association of a congenital myopathy and an atypical form of normokalaemic paralysis observed in our patient is unique or may also apply to other 
RYRI mutations. Episodes of hyperkalaemic periodic paralysis have been described previously in a genetically unresolved patient with an eye movement disorder, interpreted as supranuclear gaze palsy, but possibly caused by external ophthalmoplegia in the context of an $\mathrm{MmD}$ phenotype [30]. More recently, a causative association between periodic paralysis and a $R Y R 1$ substitution, c. $7025 \mathrm{~A}>\mathrm{G}$, was initially postulated in a patient with both MHS and periodic paralysis but later rejected because of identification of the same change in other unrelated patients with MHS but without periodic paralysis [16]. However, as RYRI screening in these patients was performed at the genomic and not muscle-derived cDNA level, potential monoallelic vs. biallelic RYRI expression in muscle potentially explaining the discrepancy between phenotypes could not therefore be excluded. "Unmasking" of mutations due to monoallelic $R Y R I$ allele expression has emerged as an important mechanism in the pathogenesis of $\mathrm{MmD}$ and ophthalmoplegia [6,8]. Some RYR1 mutations may therefore behave as dominant with regard to MHS, but recessives with regard to congenital myopathy (e.g. MmD) and possible other phenotypes (e.g. atypical periodic paralysis), an hypothesis further supported by the lack of myopathic and paralytic symptoms in the asymptomatic carrier parent of our patient.

Whilst mutations in the SCN4A and the CACNAIS gene have been firmly established as the most common cause of hyper- and hypokalaemic forms of periodic paralyses, a substantial proportion of patients presenting with classic clinical features of these conditions remain genetically unresolved. In a large cohort of 226 patients from 56 families with periodic paralysis [31], almost $40 \%$ had no confirmed $S C N 4 A$ or $C A C N A 1 S$ mutation. Interestingly, mild fixed weakness between episodes without defined myopathic changes other than the vacuolar pathology commonly observed with $C A C N A 1 S$ mutations and less frequently with $S C N 4 A$ mutations is suggestive of an associated myopathy in this subgroup of genetically unresolved cases.

Our findings indicate a new, $R Y R I$-related form of periodic paralysis and suggest the $R Y R I$ gene is a candidate for genetically unresolved cases of periodic paralysis, particularly those with symptoms divergent from genetically resolved forms of periodic paralysis and additional features of a congenital myopathy. The characteristic muscle MRI found in $R Y R I$ related diseases could be helpful in raising the possibility of involvement of the RYRI gene.

\section{Supplementary Material}

Refer to Web version on PubMed Central for supplementary material.

\section{Acknowledgments}

We would like to thank the financial support from the Muscular Dystrophy Association USA, the National Institute of Health (AR044657 to R.T.D. and AR018687 to G.M.), and the NSCAG support to the Dubowitz Neuromuscular Centre. HJ has been supported by a grant from the Guy's and St. Thomas' Charitable Foundation and REL by a National Institutes of Health Dental and Craniofacial Training Grant (T32DE07202).

\section{References}

1. Treves S, Anderson AA, Ducreux S, Divet A, Bleunven C, Grasso C, et al. Ryanodine receptor 1 mutations, dysregulation of calcium homeostasis and neuromuscular disorders. Neuromuscul Disord. 2005; 15(9-10):577-87. [PubMed: 16084090]

2. Gillard EF, Otsu K, Fujii J, Khanna VK, de Leon S, Derdemezi J, et al. A substitution of cysteine for arginine 614 in the ryanodine receptor is potentially causative of human malignant hyperthermia. Genomics. 1991; 11(3):751-5. [PubMed: 1774074] 
3. Zhang Y, Chen HS, Khanna VK, de Leon S, Phillips MS, Schappert K, et al. A mutation in the human ryanodine receptor gene associated with central core disease. Nat Genet. 1993; 5(1):46-50. [PubMed: 8220422]

4. Jungbluth H, Zhou H, Hartley L, Halliger-Keller B, Messina S, Longman C, et al. Minicore myopathy with ophthalmoplegia caused by mutations in the ryanodine receptor type 1 gene. Neurology. 2005; 65(12):1930-5. [PubMed: 16380615]

5. Jungbluth H, Zhou H, Sewry CA, Robb S, Treves S, Bitoun M, et al. Centronuclear myopathy due to a de novo dominant mutation in the skeletal muscle ryanodine receptor (RYR1) gene. Neuromuscul Disord. 2007; 17(4):338-45. [PubMed: 17376685]

6. Zhou H, Brockington M, Jungbluth H, Monk D, Stanier P, Sewry CA, et al. Epigenetic allele silencing unveils recessive RYR1 mutations in core myopathies. Am J Hum Genet. 2006; 79(5): 859-68. [PubMed: 17033962]

7. Monnier N, Ferreiro A, Marty I, Labarre-Vila A, Mezin P, Lunardi J. A homozygous splicing mutation causing a depletion of skeletal muscle RYR1 is associated with multi-minicore disease congenital myopathy with ophthalmoplegia. Hum Mol Genet. 2003; 12(10):1171-8. [PubMed: 12719381]

8. Monnier N, Marty I, Faure J, Castiglioni C, Desnuelle C, Sacconi S, et al. Null mutations causing depletion of the type 1 ryanodine receptor (RYR1) are commonly associated with recessive structural congenital myopathies with cores. Hum Mutat. 2008; 29(5):670-8. [PubMed: 18253926]

9. Fontaine B, Khurana TS, Hoffman EP, Bruns GA, Haines JL, Trofatter JA, et al. Hyperkalemic periodic paralysis and the adult muscle sodium channel alpha-subunit gene. Science. 1990; 250(4983):1000-2. [PubMed: 2173143]

10. Rojas CV, Wang JZ, Schwartz LS, Hoffman EP, Powell BR, Brown RH Jr. A Met-to-Val mutation in the skeletal muscle $\mathrm{Na}^{+}$channel alpha-subunit in hyperkalaemic periodic paralysis. Nature. 1991; 354(6352):387-9. [PubMed: 1659668]

11. Boerman RH, Ophoff RA, Links TP, van Eijk R, Sandkuijl LA, Elbaz A, et al. Mutation in DHP receptor alpha 1 subunit (CACLN1A3) gene in a Dutch family with hypokalaemic periodic paralysis. J Med Genet. 1995; 32(1):44-7. [PubMed: 7897626]

12. Murray BE, Ohlendieck K. Cross-linking analysis of the ryanodine receptor and alpha1dihydropyridine receptor in rabbit skeletal muscle triads. Biochem J. 1997; 324(Pt 2):689-96. [PubMed: 9182735]

13. Hirata H, Watanabe T, Hatakeyama J, Sprague SM, Saint-Amant L, Nagashima A, et al. Zebrafish relatively relaxed mutants have a ryanodine receptor defect, show slow swimming and provide a model of multi-minicore disease. Development. 2007; 134(15):2771-81. [PubMed: 17596281]

14. Takeshima H, Iino M, Takekura H, Nishi M, Kuno J, Minowa O, et al. Excitation-contraction uncoupling and muscular degeneration in mice lacking functional skeletal muscle ryanodinereceptor gene. Nature. 1994; 369(6481):556-9. [PubMed: 7515481]

15. Monnier N, Procaccio V, Stieglitz P, Lunardi J. Malignant-hyperthermia susceptibility is associated with a mutation of the alpha 1-subunit of the human dihydropyridine-sensitive L-type voltage-dependent calcium-channel receptor in skeletal muscle. Am J Hum Genet. 1997; 60(6): 1316-25. [PubMed: 9199552]

16. Marchant CL, Ellis FR, Halsall PJ, Hopkins PM, Robinson RL. Mutation analysis of two patients with hypokalemic periodic paralysis and suspected malignant hyperthermia. Muscle Nerve. 2004; 30(1):114-7. [PubMed: 15221887]

17. Jungbluth H, Davis MR, Muller C, Counsell S, Allsop J, Chattopadhyay A, et al. Magnetic resonance imaging of muscle in congenital myopathies associated with RYR1 mutations. Neuromuscul Disord. 2004; 14(12):785-90. [PubMed: 15564033]

18. McManis PG, Lambert EH, Daube JR. The exercise test in periodic paralysis. Muscle Nerve. 1986; 9(8):704-10. [PubMed: 3785281]

19. Ferreiro A, Quijano-Roy S, Pichereau C, Moghadaszadeh B, Goemans N, Bonnemann C, et al. Mutations of the selenoprotein $\mathrm{N}$ gene, which is implicated in rigid spine muscular dystrophy, cause the classical phenotype of multiminicore disease: reassessing the nosology of early-onset myopathies. Am J Hum Genet. 2002; 71(4):739-49. [PubMed: 12192640] 
20. Jungbluth H, Muntoni F, Ferreiro A. 150th ENMC International Workshop: Core Myopathies, 911th March 2007, Naarden, The Netherlands. Neuromuscul Disord. 2008; 18(12):989-96. [PubMed: 18948004]

21. Dubowitz, V.; Sewry, CA. Muscle biopsy - A practical approach. 3. London: WB Saunders; 2007.

22. Zhou H, Jungbluth H, Sewry CA, Feng L, Bertini E, Bushby K, et al. Molecular mechanisms and phenotypic variation in RYR1-related congenital myopathies. Brain. 2007; 130(Pt 8):2024-36. [PubMed: 17483490]

23. Zhou H, Yamaguchi N, Xu L, Wang Y, Sewry C, Jungbluth H, et al. Characterization of recessive RYR1 mutations in core myopathies. Hum Mol Genet. 2006; 15(18):2791-803. [PubMed: 16940308]

24. Meissner G. Regulation of mammalian ryanodine receptors. Front Biosci. 2002; 7:d2072-80. [PubMed: 12438018]

25. Nakai J, Dirksen RT, Nguyen HT, Pessah IN, Beam KG, Allen PD. Enhanced dihydropyridine receptor channel activity in the presence of ryanodine receptor. Nature. 1996; 380(6569):72-5. [PubMed: 8598910]

26. Lyfenko AD, Ducreux S, Wang Y, Xu L, Zorzato F, Ferreiro A, et al. Two central core disease (CCD) deletions in the C-terminal region of RYR1 alter muscle excitation-contraction (EC) coupling by distinct mechanisms. Hum Mutat. 2007; 28(1):61-8. [PubMed: 16958053]

27. Galli L, Orrico A, Cozzolino S, Pietrini V, Tegazzin V, Sorrentino V. Mutations in the RYR1 gene in Italian patients at risk for malignant hyperthermia: evidence for a cluster of novel mutations in the C-terminal region. Cell Calcium. 2002; 32(3):143-51. [PubMed: 12208234]

28. Wu S, Ibarra MC, Malicdan MC, Murayama K, Ichihara Y, Kikuchi H, et al. Central core disease is due to RYR1 mutations in more than $90 \%$ of patients. Brain. 2006; 129(Pt 6):1470-80. [PubMed: 16621918]

29. Mackrill JJ. Possible regulation of the skeletal muscle ryanodine receptor by a polyubiquitin binding subunit of the 26S proteasome. Biochem Biophys Res Commun. 1998; 245(2):428-9. [PubMed: 9571168]

30. Foster NL. Progressive supranuclear palsy and hyperkalemic periodic paralysis. Arch Neurol. 1980; 37(7):461-2. [PubMed: 7387497]

31. Miller TM, Dias da Silva MR, Miller HA, Kwiecinski H, Mendell JR, Tawil R, et al. Correlating phenotype and genotype in the periodic paralyses. Neurology. 2004; 63(9):1647-55. [PubMed: 15534250]

\section{Appendix A. Supplementary data}

Supplementary data associated with this article can be found, in the online version, at doi: 10.1016/j.nmd.2009.12.005. 

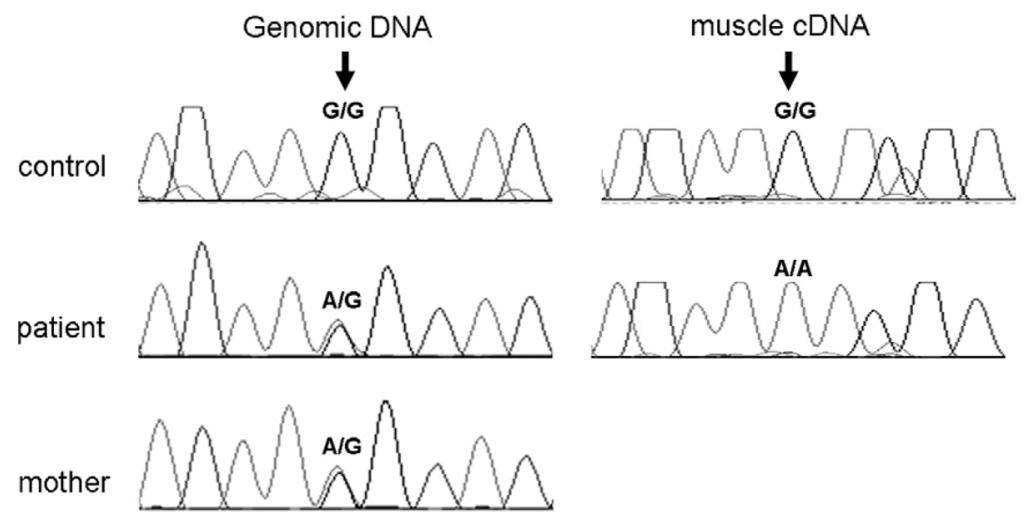

Fig. 1.

Chromatograms showing expression of the c.8816G $>$ A substitution in genomic DNA and skeletal muscle-derived cDNA. In the patient the substitution is heterozygous $(\mathrm{A} / \mathrm{G})$ at the genomic DNA level but homozygous at the transcriptional level (A), suggesting that the wild type allele (carrying nucleotide $G$ ) is not transcribed in the patient's skeletal muscle. The c.8816G>A mutation was maternally inherited. Since the change in this patient affected the last nucleotide residue in exon 57, the sequences following this residue differed between DNA and cDNA. 


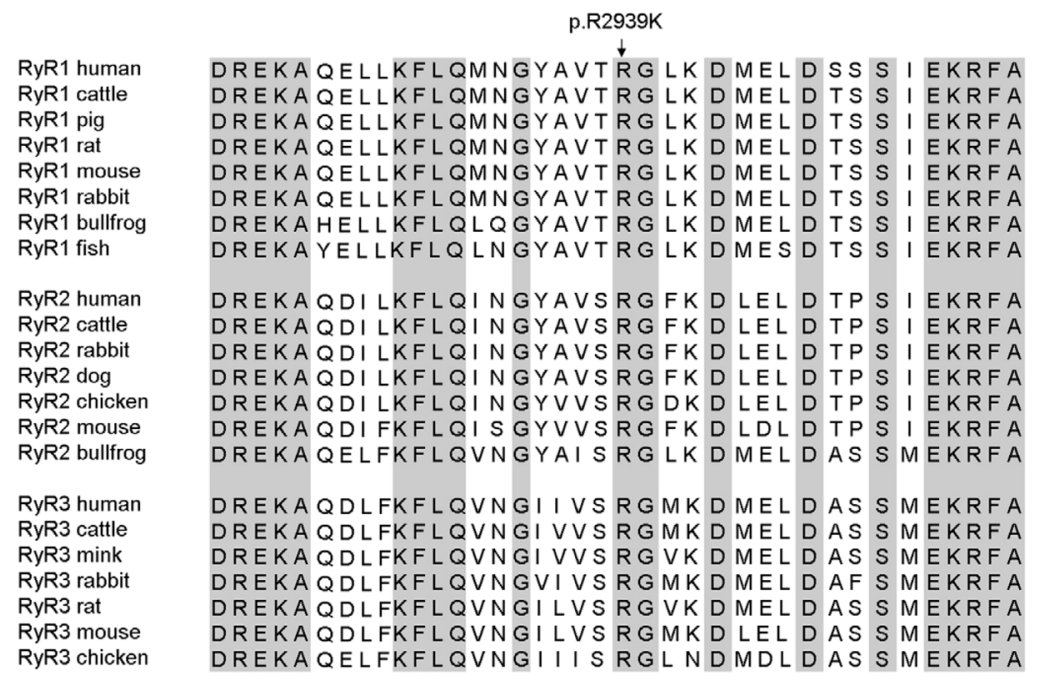

Fig. 2.

Amino acid sequence alignment of the residues flanking the mutated arginine residue at position 2939 in RyR1. Residues conserved across different RyR isoforms and species are shaded. The substituted arginine residue is strictly conserved in all three RyR isoforms and across all species. 
A

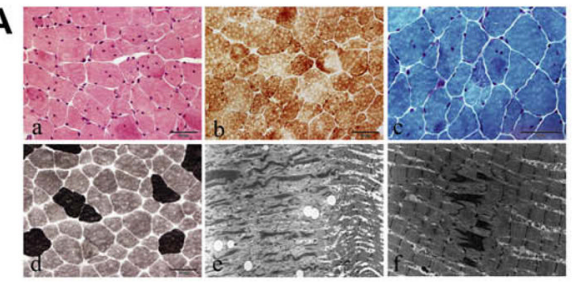

B

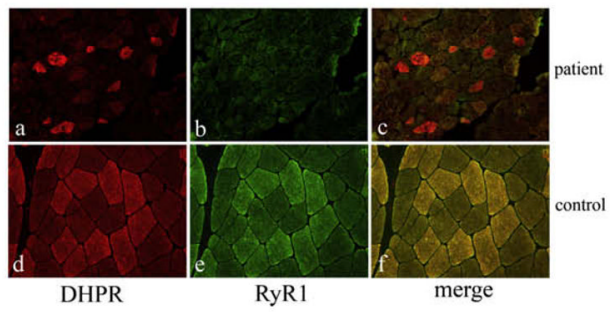

C

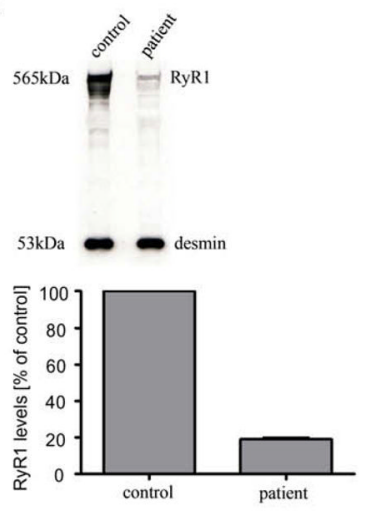

Fig. 3.

Analysis of muscle biopsies. (A) Histochemical and electron microscopic analysis of transverse sections obtained from a biopsy of the patient's quadriceps muscle. H\&E (a) and Gomori trichrome (c) stains show marked variability in fibre size with increased internal nucleation in the absence of excess connective or fatty tissue. ATPase stain pre-incubated at pH 9.4 (d) and cytochrome oxidase (COX) stain (b) are consistent with marked type 1 fiber predominance. Uneven COX staining reveals core-like structures in some fibres. Electron microscopy (e \& f) demonstrates variable degrees of sarcomeric disorganization, ranging from Z-band streaming to more advanced disarray of myofibrils. (B) Immunohistochemical distribution of DHPR and RyR1 protein in transverse sections obtained in the patient (a-c) and a normal control (d-f). In most fibres, reduced RyR1 immunofluorescence as well as the aggregation of DHPR in some fibres are observed in the patient compared to normal control (c \& f). (C) Western blot analysis of skeletal muscle tissue. Protein extracted from patient's muscle biopsy shows a significant reduction of RyR1 expression $(565 \mathrm{kDa})$ compared to the control sample. Desmin immunoreactivity was used as a muscle-specific internal control. Semi-quantification by using densitometric analyses showed that in the patient there was only about $20 \%$ residual RyR1 protein expressed (result expressed as percentage of control). 

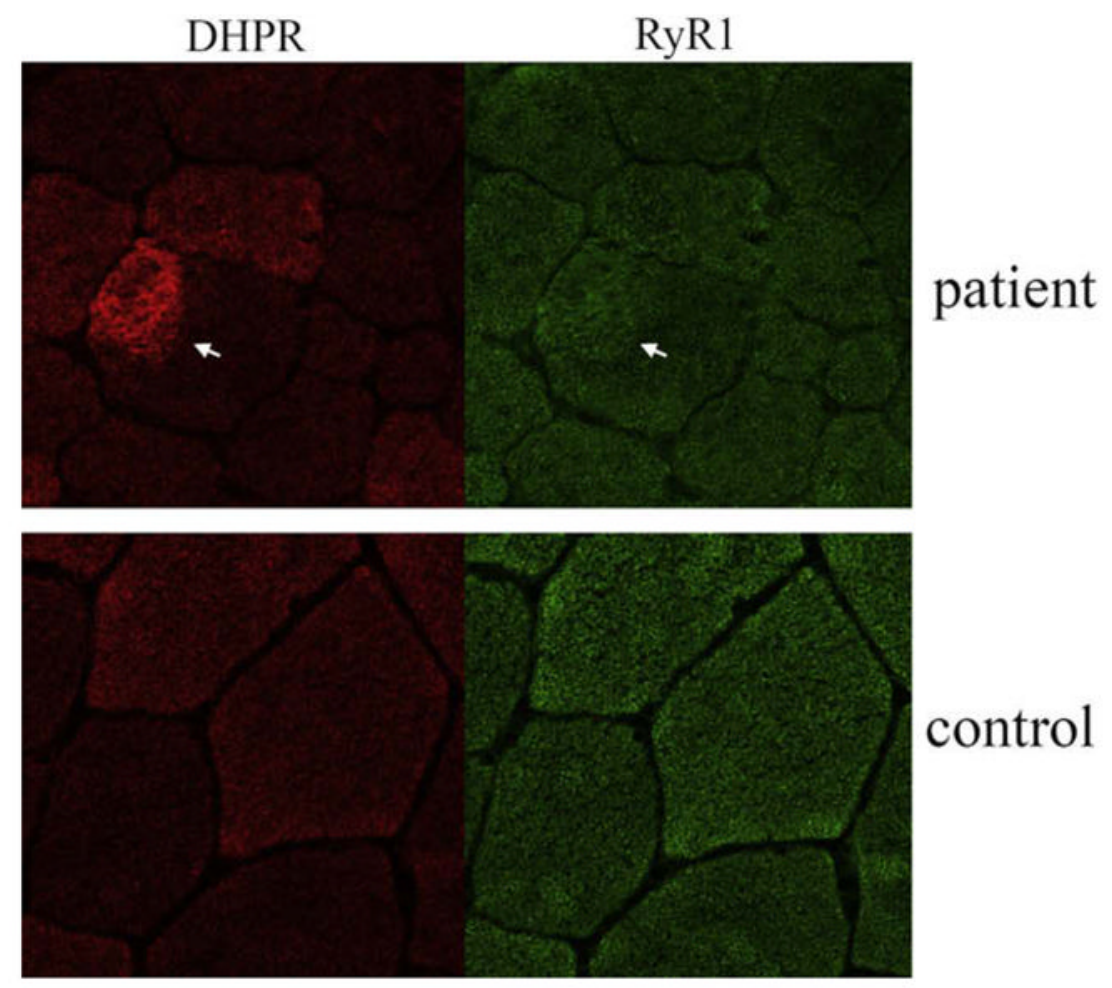

Fig. 4.

Double staining sections were examined by confocal microscope at high magnification (40x objective). Compared to control biopsy, patient's muscle fibre showed abnormal accumulation of DHPR protein and slight reduction of RyR1 antibody staining in the corresponding area (arrows). 

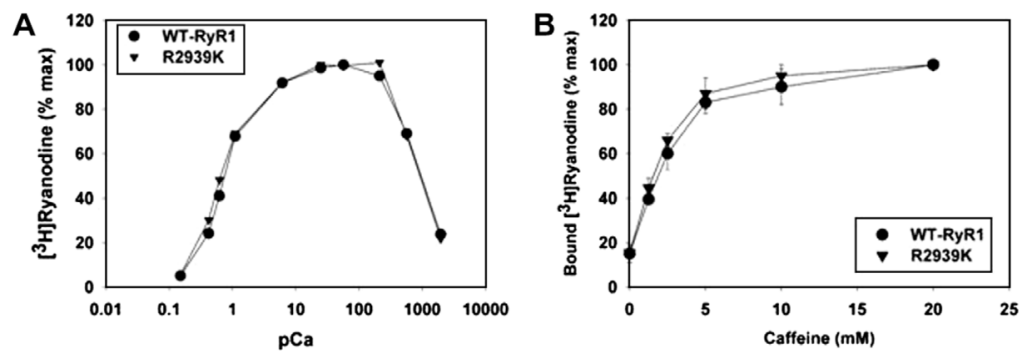

Fig. 5.

Calcium (A) and caffeine (B) dependence of $\left[{ }^{3} \mathrm{H}\right]$ ryanodine binding to recombinant WT RyR1 and the R2939K RyR1 mutant. Specific $\left[{ }^{3} \mathrm{H}\right]$ ryanodine binding to crude membrane fractions containing WT or R2939K mutant RyR1 was determined as described in Methods. Data represent the mean of 3 independent experiments. There is no difference in $\mathrm{Ca}^{2+}$ or caffeine dependence of $\left[{ }^{3} \mathrm{H}\right]$ ryanodine binding to WT RyR1 (closed circles) and R2939K mutant RyR1 (closed triangles). 
A
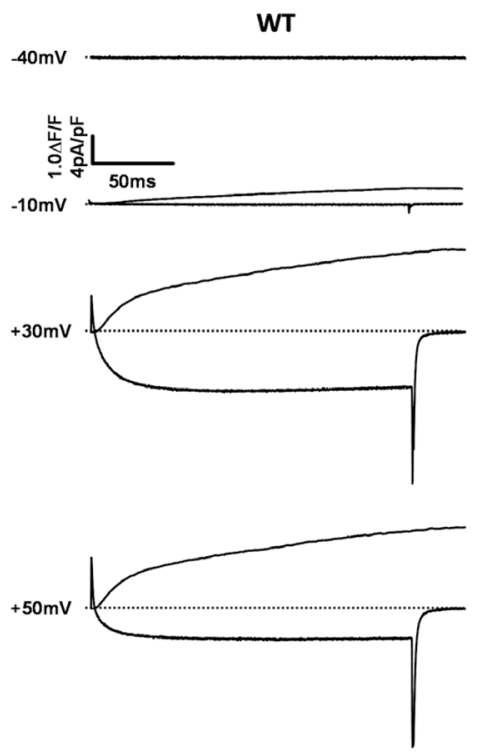

B

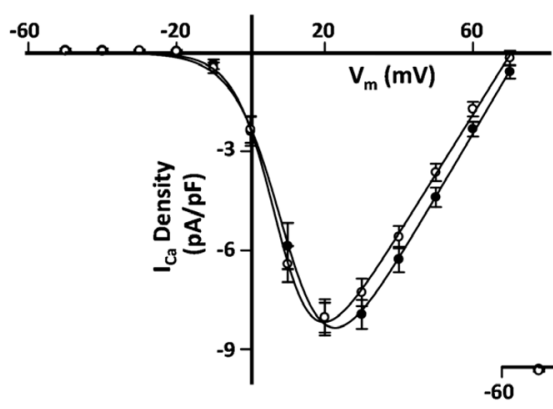

RK
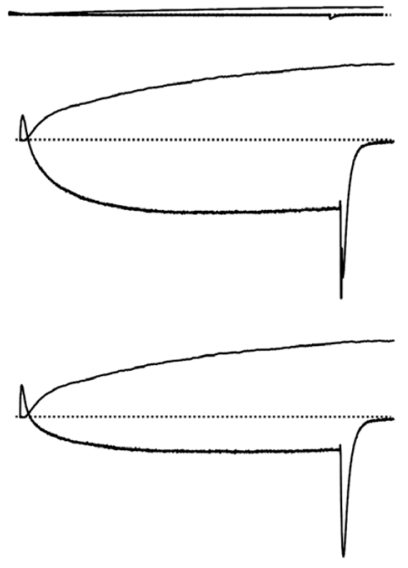

C

Fig. 6.

Comparison of DHPR-RyR1 coupling in dyspedic myotubes expressing either wild-type (WT) RyR1 or R2939K (RK). A: Representative voltage-gated $\mathrm{Ca}^{2+}$ transients (upper traces) and L-type $\mathrm{Ca}^{2+}$ currents (lower traces) recorded in response to $200 \mathrm{~ms}$ depolarizing pulses to the indicated membrane potentials from WT-(left) and R2939Kexpressing (right) dyspedic myotubes. B: Average ( \pm SEM) I-V curves for dyspedic myotubes expressing WT RYR1 (closed circles; $n=21$ ) and RyR1-R2939K (open circles; $n=21$ ). The average values for the parameters obtained by separately fitting each myotube within a group are given in Supplementary Table S1. C: Average voltage dependence of SR $\mathrm{Ca}^{2+}$ release for dyspedic myotubes expressing WT-(closed circles; n=21) and R2939K-expressing (open circles; $\mathrm{n}=21)$ dyspedic myotubes. The average values $( \pm \mathrm{SEM})$ for the parameters obtained by separately fitting each myotube within a group are given in Supplementary Table S1. 